\title{
The expected values of Kirchhoff indices in the random polyphenyl and spiro chains*
}

\author{
Guihua Huang \\ College of Mathematics and Computer Science, Hunan Normal University, \\ Changsha, Hunan 410081, P. R. China \\ Meijun Kuang \\ College of Mathematics and Computer Science, Hunan Normal University, \\ Changsha, Hunan 410081, P. R. China \\ Hanyuan Deng ${ }^{\dagger}$ \\ College of Mathematics and Computer Science, Hunan Normal University, \\ Changsha, Hunan 410081, P. R. China
}

Received 14 March 2013, accepted 22 April 2014, published online 28 November 2014

\begin{abstract}
The Kirchhoff index $K f(G)$ of a graph $G$ is the sum of resistance distances between all pairs of vertices in $G$. In this paper, we obtain exact formulas for the expected values of the Kirchhoff indices of the random polyphenyl and spiro chains, which are graphs of a class of unbranched multispiro molecules and polycyclic aromatic hydrocarbons. Moreover, we obtain a relation between the expected values of the Kirchhoff indices of a random polyphenyl and its random hexagonal squeeze, and the average values for the Kirchhoff indices of all polyphenyl chains and all spiro chains with $n$ hexagons, respectively.

Keywords: Expected value, average value, Kirchhoff index, resistance distance, polyphenyl chain, spiro chain.
\end{abstract}

Math. Subj. Class.: 05C12, 05C80, 05C90, 05D40

\footnotetext{
* Project supported by Hunan Provincial Natural Science Foundation of China(13JJ3053).

${ }^{\dagger}$ Corresponding Author.

E-mail addresses: 380026412@qq.com (Guihua Huang), 1075998525@qq.com (Meijun Kuang), hydeng@hunnu.edu.cn (Hanyuan Deng)
}

(a)(i) This work is licensed under http://creativecommons.org/licenses/by/3.0/ 


\section{Introduction}

Based on the electrical network theory, Klein and Randic [13] introduced the concept of resistance distance. A connected graph $G$ with vertex set $\left\{v_{1}, v_{2}, \cdots, v_{n}\right\}$ is viewed as an electrical network $N$ by replacing each edge of $G$ with a unit resistor, the resistance distance between $v_{i}$ and $v_{j}$, denoted by $r_{G}\left(v_{i}, v_{j}\right)$ or $r\left(v_{i}, v_{j}\right)$, is the elective resistance between them as computed by the methods of the theory of resistive electrical networks based on Ohm's and Kirchhoff's laws in $N$.

The Kirchhoff index of $G$, denoted by $K f(G)$, is the sum of resistance distances between all pairs of vertices in $G$, namely,

$$
K f(G)=\sum_{i<j} r_{G}\left(v_{i}, v_{j}\right)
$$

Like many topological indices, Kirchhoff index is a structure descriptor. The resistance distance is also intrinsic to the graph with some nice purely mathematical and physical interpretations [14] [15]. Also, the Kirchhoff index has been found very useful in chemistry, such as in assessing cyclicity of polycyclic structures including fullerenes, linear hexagonal chains and some special molecular graphs such as circulant graphs, distance-regular graphs and Möbius ladders [1] [18] [22] [24]. Bonchev et al. [4] used it in polymer science and found that the Kirchhoff index in their approach is especially useful for defining the topological radius $R_{t o p}=\frac{K f}{n^{2}}$ of macromolecules containing cyclic fragments. Some closed-form formulae for Kirchhoff index have been given for circulant graphs, linear hexagonal chains and so on [1] [16] [19] [22]. The resistance distance is also well studied in mathematical literatures. Much work has been done to compute Kirchhoff index of some classes of graphs, or give some bounds for Kirchhoff index of graphs and characterize extremal graphs. For instance, unicyclic and bicyclic graphs with extremal Kirchhoff index are characterized and sharp bounds for Kirchhoff index of such graphs are obtained [6] [12] [21] [25] [26].

Polyphenyls and their derivatives, which can be used in organic synthesis, drug synthesis, heat exchangers, etc., attracted the attention of chemists for many years [11] [17] [20]. Spiro compounds are an important class of cycloalkanes in organic chemistry. A spiro union in spiro compounds is a linkage between two rings that consists of a single atom common to both rings and a free spiro union is a linkage that consists of the only direct union between the rings. Some results on energy, Merrifield-Simmons index, Hosoya index and Wiener index of the spiro and polyphenyl chains were reported in [2] [5] [9] [27]. Recently, Deng [7] [8] [10] gave the recurrences or explicit formulae for computing the Wiener index and Kirchhoff index of spiro and polyphenyl chains. Yang and Zhang [23] obtained a simple exact formula for the expected value of the Wiener index of a random polyphenyl chain. In this paper, we will consider the expected values of the Kirchhoff index of random polyphenyl and spiro chains.

A polyphenyl chain $P P C_{n}$ with $n$ hexagons can be regarded as a polyphenyl chain $P P C_{n-1}$ with $n-1$ hexagons to which a new terminal hexagon has been adjoined by a cut edge, see Figure 1.

Let $P P C_{n}=H_{1} H_{2} \cdots H_{n}$ be a polyphenyl chain with $n(n \geq 2)$ hexagons, where $H_{k}$ is the $k$-th hexagon of $P P C_{n}$ attached to $H_{k-1}$ by a cut edge $u_{k-1} c_{k}, k=2,3, \cdots, n$. A vertex $v$ of $H_{k}$ is said to be ortho-, meta- and para-vertex of $H_{k}$ if the distance between $v$ and $c_{k}$ is 1,2 and 3, denoted by $o_{k}, m_{k}$ and $p_{k}$, respectively. Examples of ortho-, meta-, and 
para-vertices are shown in Figure 1. Except the first hexagon, any hexagon in a polyphenyl chain has two ortho-vertices, two meta-vertices and one para-vertex.

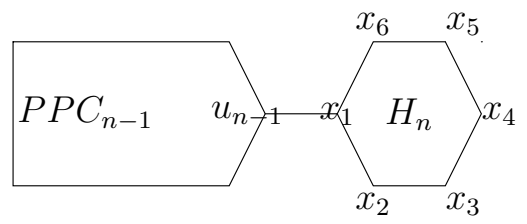

Figure 1: A polyphenyl chain $P P C_{n}$ with $n$ hexagons, $c_{n}=x_{1}$ and ortho-vertices $o_{n}=x_{2}, x_{6}$, meta-vertices $m_{n}=x_{3}, x_{5}$, and para-vertex $p_{n}=x_{4}$ in $H_{n}$.

A polyphenyl chain $P P C_{n}$ is a polyphenyl ortho-chain if $u_{k}=o_{k}$ for $2 \leq k \leq n-1$. A polyphenyl chain $P P C_{n}$ is a polyphenyl meta-chain if $u_{k}=m_{k}$ for $2 \leq k \leq n-1$. A polyphenyl chain $P P C_{n}$ is a polyphenyl para-chain if $u_{k}=p_{k}$ for $2 \leq k \leq n-1$. The polyphenyl ortho-, meta- and para-chain with $n$ hexagons are denoted by $\overline{O_{n}}, \overline{M_{n}}$ and $\overline{P_{n}}$, respectively.

For $n \geq 3$, the terminal hexagon can be attached to meta-, ortho-, or para-vertex in three ways, which results in the local arrangements we describe as $P P C_{n+1}^{1}, P P C_{n+1}^{2}$, $P P C_{n+1}^{3}$, see Figure 2 .

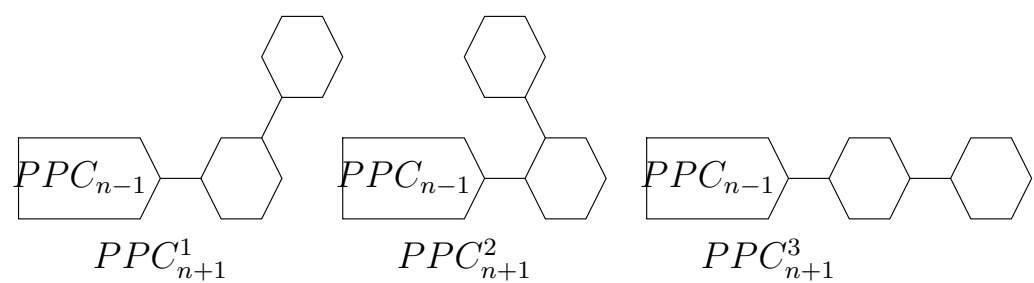

Figure 2: The three types of local arrangements in polyphenyl chains.

A random polyphenyl chain $\operatorname{PPC}\left(n, p_{1}, p_{2}\right)$ with $n$ hexagons is a polyphenyl chain obtained by stepwise addition of terminal hexagons. At each step $k(=3,4, \cdots, n)$, a random selection is made from one of the three possible constructions:

(i) $P P C_{k-1} \rightarrow P P C_{k}^{1}$ with probability $p_{1}$,

(ii) $P P C_{k-1} \rightarrow P P C_{k}^{2}$ with probability $p_{2}$,

(iii) $P P C_{k-1} \rightarrow P P C_{k}^{3}$ with probability $1-p_{1}-p_{2}$

where the probabilities $p_{1}$ and $p_{2}$ are constants, irrespective to the step parameter $k$.

Specially, the random polyphenyl chain $\operatorname{PPC}(n, 1,0)$ is the polyphenyl meta-chain $\overline{M_{n}}, \operatorname{PPC}(n, 0,1)$ is the polyphenyl orth-chain $\overline{O_{n}}$, and $P P C(n, 0,0)$ is the polyphenyl para-chain $\overline{P_{n}}$, respectively.

Also, a spiro chain $S P C_{n}$ with $n$ hexagons can be regarded as a spiro chain $S P C_{n-1}$ with $n-1$ hexagons to which a new terminal hexagon has been adjoined, see Figure 3 . 


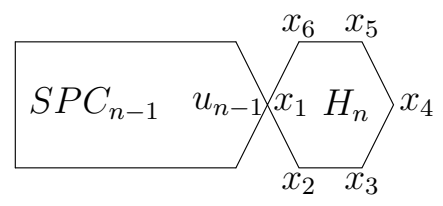

Figure 3: A spiro chain $S P C_{n}$ with $n$ hexagons.

For $n \geq 3$, the terminal hexagon can also be attached in three ways, which results in the local arrangements we describe as $S P C_{n+1}^{1}, S P C_{n+1}^{2}, S P C_{n+1}^{3}$, see Figure 4.
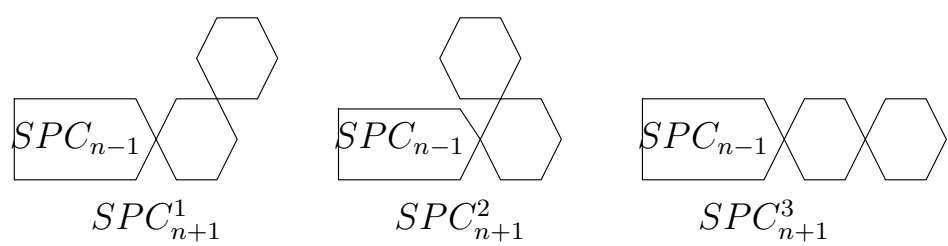

Figure 4: The three types of local arrangements in spiro chains.

A random spiro chain $S P C\left(n, p_{1}, p_{2}\right)$ with $n$ hexagons is a spiro chain obtained by stepwise addition of terminal hexagons. At each step $k(=3,4, \cdots, n)$, a random selection is made from one of the three possible constructions:

(i) $S P C_{k-1} \rightarrow S P C_{k}^{1}$ with probability $p_{1}$,

(ii) $S P C_{k-1} \rightarrow S P C_{k}^{2}$ with probability $p_{2}$,

(iii) $S P C_{k-1} \rightarrow S P C_{k}^{3}$ with probability $1-p_{1}-p_{2}$

where the probabilities $p_{1}$ and $p_{2}$ are constants, irrelative to the step parameter $k$.

Similarly, the random spiro chain $S P C(n, 1,0), P P C(n, 0,1)$ and $P P C(n, 0,0)$ are the spiro meta-chain $M_{n}$, the spiro orth-chain $O_{n}$ and the spiro para-chain $P_{n}$, respectively.

For a random polyphenyl chain $\operatorname{PPC}\left(n, p_{1}, p_{2}\right)$ and a random spiro chain $S P C(n$, $\left.p_{1}, p_{2}\right)$, their Kirchhoff indices are random variables. In this paper, we will obtain exact formulas for the expected values $E\left(K f\left(P P C\left(n, p_{1}, p_{2}\right)\right)\right)$ and $E\left(K f\left(S P C\left(n, p_{1}, p_{2}\right)\right)\right)$ of the Kirchhoff indices of random polyphenyl and spiro chains, respectively.

\section{Main results}

\subsection{The Kirchhoff index of the random polyphenyl chain}

In this section, we will consider the Kirchhoff index of the random polyphenyl chain.

Theorem 2.1. For $n \geq 1$, the expected value of the Kirchhoff index of the random polyphenyl chain $P P C\left(n, p_{1}, p_{2}\right)$ is

$E\left(K f\left(P P C\left(n, p_{1}, p_{2}\right)\right)\right)=\left(15-p_{1}-4 p_{2}\right) n^{3}+\left(3 p_{1}+12 p_{2}+8\right) n^{2}-\left(2 p_{1}+8 p_{2}+\frac{11}{2}\right) n$

Proof. Note that the polyphenyl chain $P P C_{n}$ is obtained by attaching $P P C_{n-1}$ a new terminal hexagon by an edge, we suppose that the terminal hexagon is spanned by vertices $x_{1}, x_{2}, x_{3}, x_{4}, x_{5}, x_{6}$, and the new edge is $u_{n-1} x_{1}$ (see Fig.1). Then

(i) For any $v \in P P C_{n-1}$, 


$$
\begin{aligned}
& r\left(x_{1}, v\right)=r\left(u_{n-1}, v\right)+1, r\left(x_{2}, v\right)=r\left(u_{n-1}, v\right)+1+\frac{5}{6}, \\
& r\left(x_{3}, v\right)=r\left(u_{n-1}, v\right)+1+\frac{4}{3}, r\left(x_{4}, v\right)=r\left(u_{n-1}, v\right)+1+\frac{3}{2}, \\
& r\left(x_{5}, v\right)=r\left(u_{n-1}, v\right)+1+\frac{4}{3}, r\left(x_{6}, v\right)=r\left(u_{n-1}, v\right)+1+\frac{5}{6}
\end{aligned}
$$

(ii) $P P C_{n-1}$ has $6(n-1)$ vertices;

(iii) For $k \in\{1,2,3,4,5,6\}, \sum_{i=1}^{6} r\left(x_{k}, x_{i}\right)=\frac{35}{6}$.

So, we have

$$
\begin{aligned}
& r\left(x_{1} \mid P P C_{n}\right)=r\left(u_{n-1} \mid P P C_{n-1}\right)+1 \times 6(n-1)+\frac{35}{6} \\
& r\left(x_{2} \mid P P C_{n}\right)=r\left(u_{n-1} \mid P P C_{n-1}\right)+\left(1+\frac{5}{6}\right) \times 6(n-1)+\frac{35}{6} \\
& r\left(x_{3} \mid P P C_{n}\right)=r\left(u_{n-1} \mid P P C_{n-1}\right)+\left(1+\frac{4}{3}\right) \times 6(n-1)+\frac{35}{6} \\
& r\left(x_{4} \mid P P C_{n}\right)=r\left(u_{n-1} \mid P P C_{n-1}\right)+\left(1+\frac{3}{2}\right) \times 6(n-1)+\frac{35}{6} \\
& r\left(x_{5} \mid P P C_{n}\right)=r\left(x_{3} \mid P P C_{n-1}\right) \\
& r\left(x_{6} \mid P P C_{n}\right)=r\left(x_{2} \mid P P C_{n-1}\right) \\
& \text { where } r(x \mid G)=\sum_{y \in V(G)} r(x, y), \text { and }
\end{aligned}
$$

$$
\begin{aligned}
K f\left(P P C_{n}\right) & =K f\left(P P C_{n-1}\right)+6 r\left(u_{n-1} \mid P P C_{n-1}\right)+71 n-36-\frac{1}{2} \sum_{i=1}^{6} \sum_{j=1}^{6} r\left(v_{i}, v_{j}\right) \\
& =K f\left(P P C_{n-1}\right)+6 r\left(u_{n-1} \mid P P C_{n-1}\right)+71 n-36-\frac{35}{2}
\end{aligned}
$$

Then

$$
K f\left(P P C_{n+1}\right)=K f\left(P P C_{n}\right)+6 r\left(u_{n} \mid P P C_{n}\right)+71 n+\frac{35}{2}
$$

For a random polyphenyl chain $P P C\left(n, p_{1}, p_{2}\right)$, the resistance number $r\left(u_{n} \mid P P C(n\right.$, $\left.\left.p_{1}, p_{2}\right)\right)$ is a random variable, and its expected value is denoted by

$$
U_{n}=E\left(r\left(u_{n} \mid P P C\left(n, p_{1}, p_{2}\right)\right)\right)
$$

By the expectation operator and (1), we can obtain a recursive relation for the expected value of the Kirchhoff number of a random polyphenyl chain $\operatorname{PPC}\left(n, p_{1}, p_{2}\right)$

$$
E\left(K f\left(P P C\left(n+1, p_{1}, p_{2}\right)\right)=E\left(K f\left(P P C\left(n, p_{1}, p_{2}\right)\right)\right)+6 U_{n}+71 n+\frac{35}{2}\right.
$$

Now, we consider computing $U_{n}$.

(i) If $P P C_{n} \rightarrow P P C_{n+1}^{1}$ with probability $p_{1}$, then $u_{n}$ coincides with the vertex $x_{3}$ or $x_{5}$. Consequently, $r\left(u_{n} \mid P P C_{n}\right)$ is given by $r\left(x_{3} \mid P P C_{n}\right)$ with probability $p_{1}$.

(ii) If $P P C_{n} \rightarrow P P C_{n+1}^{2}$ with probability $p_{2}$, then $u_{n}$ coincides with the vertex $x_{2}$ or $x_{6}$. Consequently, $r\left(u_{n} \mid P P C_{n}\right)$ is given $r\left(x_{2} \mid P P C_{n}\right)$ with probability $p_{2}$.

(iii) If $P P C_{n} \rightarrow P P C_{n+1}^{3}$ with probability $1-p_{1}-p_{2}$, then $u_{n}$ coincides with the vertex $x_{4}$. Consequently, $r\left(u_{n} \mid P P C_{n}\right)$ is given by $r\left(x_{4} \mid P P C_{n}\right)$ with probability $1-p_{1}-$ $p_{2}$. 
From (i)-(iii) above, we immediately obtain

$$
\begin{aligned}
U_{n}= & r\left(x_{3} \mid P P C_{n}\right) p_{1}+r\left(x_{2} \mid P P C_{n}\right) p_{2}+r\left(x_{4} \mid P P C_{n}\right)\left(1-p_{1}-p_{2}\right) \\
= & p_{1}\left[r\left(u_{n-1} \mid P P C\left(n-1, p_{1}, p_{2}\right)\right)+14(n-1)+\frac{35}{6}\right] \\
& +p_{2}\left[r\left(u_{n-1} \mid P P C\left(n-1, p_{1}, p_{2}\right)\right)+11(n-1)+\frac{35}{6}\right] \\
& +\left(1-p_{1}-p_{2}\right)\left[r\left(u_{n-1} \mid P P C\left(n-1, p_{1}, p_{2}\right)\right)+15(n-1)+\frac{35}{6}\right]
\end{aligned}
$$

By applying the expectation operator to the above equation, we obtain

$$
U_{n}=U_{n-1}+\left(15-p_{1}-4 p_{2}\right) n+p_{1}+4 p_{2}-\frac{55}{6}
$$

And $U_{1}=E\left(r\left(u_{1} \mid P P C\left(1, p_{1}, p_{2}\right)\right)\right)=\frac{35}{6}$, using the above recurrence relation, we have

$$
U_{n}=\frac{\left(15-p_{1}-4 p_{2}\right)}{2} n^{2}+\left(\frac{p_{1}}{2}+2 p_{2}-\frac{5}{3}\right) n
$$

From (2),

$$
\begin{aligned}
& E\left(K f\left(P P C\left(n+1, p_{1}, p_{2}\right)\right)\right. \\
& =E\left(K f\left(P P C\left(n, p_{1}, p_{2}\right)\right)\right)+6\left[\frac{\left(15-p_{1}-4 p_{2}\right)}{2} n^{2}+\left(\frac{p_{1}}{2}+2 p_{2}-\frac{5}{3}\right) n\right]+71 n+\frac{35}{2} \\
& =E\left(K f\left(P P C\left(n, p_{1}, p_{2}\right)\right)\right)+\left(45-3 p_{1}-12 p_{2}\right) n^{2}+\left(3 p_{1}+12 p_{2}+61\right) n+\frac{35}{2}
\end{aligned}
$$

and $E\left(K f\left(P P C\left(1, p_{1}, p_{2}\right)\right)\right)=\frac{35}{2}$.

Using the above recurrence relation, we have

$E\left(K f\left(P P C\left(n, p_{1}, p_{2}\right)\right)\right)=\left(15-p_{1}-4 p_{2}\right) n^{3}+\left(3 p_{1}+12 p_{2}+8\right) n^{2}-\left(2 p_{1}+8 p_{2}+\frac{11}{2}\right) n$

Specially, by taking $\left(p_{1}, p_{2}\right)=(1,0),(0,1)$ or $(0,0)$, respectively, and Theorem 2.1, we have

Corollary 2.2. ([8]) The Kirchhoff indices of the polyphenyl meta-chain $\overline{M_{n}}$, the polyphenyl ortho-chain $\overline{O_{n}}$ and the polyphenyl para-chain $\overline{P_{n}}$ are

$$
\begin{aligned}
& K f\left(\overline{M_{n}}\right)=14 n^{3}+11 n^{2}-\frac{15}{2} n \\
& K f\left(\overline{O_{n}}\right)=11 n^{3}+20 n^{2}-\frac{27}{2} n \\
& K f\left(\overline{P_{n}}\right)=15 n^{3}+8 n^{2}-\frac{11}{2} n
\end{aligned}
$$




\subsection{The Kirchhoff index of the random spiro chain}

In this section, we will consider the Kirchhoff index of the random spiro chain.

Theorem 2.3. For $n \geq 1$, the expected value of the Kirchhoff index of the random spiro chain $\operatorname{SPC}\left(n, p_{1}, p_{2}\right)$ is

$$
\begin{array}{r}
E\left(K f\left(S P C\left(n, p_{1}, p_{2}\right)\right)\right)=\left(\frac{25}{4}-\frac{25}{36} p_{1}-\frac{25}{9} p_{2}\right) n^{3}+\left(\frac{25}{12} p_{1}+\frac{25}{3} p_{2}+\frac{125}{12}\right) n^{2} \\
-\left(\frac{25}{18} p_{1}+\frac{50}{9} p_{2}-\frac{5}{6}\right) n .
\end{array}
$$

Proof. Note that the spiro chain $S P C_{n}$ is obtained by attaching $S P C_{n-1}$ a new terminal hexagon, we suppose that the terminal hexagon is spanned by vertices $x_{1}, x_{2}, x_{3}, x_{4}, x_{5}$, $x_{6}$, and the vertex $x_{1}$ is $u_{n-1}$ (see Fig.3). Then

(i) For any $v \in S P C_{n-1}$,

$$
\begin{aligned}
& r\left(x_{1}, v\right)=r\left(u_{n-1}, v\right), r\left(x_{2}, v\right)=r\left(u_{n-1}, v\right)+\frac{5}{6}, \\
& r\left(x_{3}, v\right)=r\left(u_{n-1}, v\right)+\frac{4}{3}, r\left(x_{4}, v\right)=r\left(u_{n-1}, v\right)+\frac{3}{2}, \\
& r\left(x_{5}, v\right)=r\left(u_{n-1}, v\right)+\frac{4}{3}, r\left(x_{6}, v\right)=r\left(u_{n-1}, v\right)+\frac{5}{6}
\end{aligned}
$$

(ii) $S P C_{n-1}$ has $5(n-1)+1$ vertices;

(iii) For $k \in\{1,2,3,4,5,6\}, \sum_{i=1}^{6} r\left(x_{k}, x_{i}\right)=\frac{35}{6}$.

So, we have

$$
\begin{gathered}
r\left(x_{1} \mid S P C_{n}\right)=r\left(u_{n-1} \mid S P C_{n-1}\right)+\frac{35}{6} \\
r\left(x_{2} \mid S P C_{n}\right)=r\left(u_{n-1} \mid S P C_{n-1}\right)+\frac{5}{6} \times(5 n-4)+\frac{5}{6}+\frac{4}{3}+\frac{3}{2}+\frac{4}{3}=r\left(u_{n-1} \mid S P C_{n-1}\right)+ \\
\frac{25}{6} \times(n-1)+\frac{35}{6} \\
r\left(x_{3} \mid S P C_{n}\right)=r\left(u_{n-1} \mid S P C_{n-1}\right)+\frac{20}{3} \times(n-1)+\frac{35}{6} \\
r\left(x_{4} \mid S P C_{n}\right)=r\left(u_{n-1} \mid S P C_{n-1}\right)+\frac{15}{2} \times(n-1)+\frac{35}{6} \\
r\left(x_{5} \mid S P C_{n}\right)=r\left(x_{3} \mid S P C_{n-1}\right) \\
r\left(x_{6} \mid S P C_{n}\right)=r\left(x_{2} \mid S P C_{n-1}\right) \\
\text { where } r(x \mid G)=\sum_{y \in V(G)} r(x, y), \text { and } \\
\qquad K f\left(S P C_{n}\right)=K f\left(S P C_{n-1}\right)+5 r\left(u_{n-1} \mid S P C_{n-1}\right)+ \\
\quad \frac{175(n-1)}{6}+35-\frac{1}{2} \sum_{i=1}^{6} \sum_{j=1}^{6} r\left(v_{i}, v_{j}\right) \\
=K f\left(S P C_{n-1}\right)+5 r\left(u_{n-1} \mid S P C_{n-1}\right)+\frac{175 n}{6}-\frac{35}{3}
\end{gathered}
$$

Then

$$
K f\left(S P C_{n+1}\right)=K f\left(S P C_{n}\right)+5 r\left(u_{n} \mid S P C_{n}\right)+\frac{175 n}{6}+\frac{35}{2}
$$

For a random spiro chain $S P C\left(n, p_{1}, p_{2}\right)$, the resistance number $r\left(u_{n} \mid S P C\left(n, p_{1}, p_{2}\right)\right)$ is a random variable, and its expected value is denoted by

$$
U_{n}=E\left(r\left(u_{n} \mid S P C\left(n, p_{1}, p_{2}\right)\right)\right) .
$$

By the expectation operator and (3), we can obtain a recursive relation for the expected value of the Kirchhoff number of a random spiro chain $\operatorname{SPC}\left(n, p_{1}, p_{2}\right)$

$$
E\left(K f\left(S P C\left(n+1, p_{1}, p_{2}\right)\right)=E\left(K f\left(S P C\left(n, p_{1}, p_{2}\right)\right)\right)+5 U_{n}+\frac{175 n}{6}+\frac{35}{2}\right.
$$


Now, we consider computing $U_{n}$.

(i) If $S P C_{n} \rightarrow S P C_{n+1}^{1}$ with probability $p_{1}$, then $u_{n}$ is the vertex $x_{3}$ or $x_{5}$. Consequently, $r\left(u_{n} \mid S P C_{n}\right)$ is given by $r\left(x_{3} \mid S P C_{n}\right)$ with probability $p_{1}$.

(ii) If $S P C_{n} \rightarrow S P C_{n+1}^{2}$ with probability $p_{2}$, then $u_{n}$ is the vertex $x_{2}$ or $x_{6}$. Consequently, $r\left(u_{n} \mid S P C_{n}\right)$ is given $r\left(x_{2} \mid S P C_{n}\right)$ with probability $p_{2}$.

(iii) If $S P C_{n} \rightarrow S P C_{n+1}^{3}$ with probability $1-p_{1}-p_{2}$, then $u_{n}$ is the vertex $x_{4}$. Consequently, $r\left(u_{n} \mid S P C_{n}\right)$ is given by $r\left(x_{4} \mid S P C_{n}\right)$ with probability $1-p_{1}-p_{2}$.

From (i)-(iii) above, we immediately obtain

$$
\begin{aligned}
U_{n}= & r\left(x_{3} \mid S P C_{n}\right) p_{1}+r\left(x_{2} \mid S P C_{n}\right) p_{2}+r\left(x_{4} \mid S P C_{n}\right)\left(1-p_{1}-p_{2}\right) \\
= & p_{1}\left[r\left(u_{n-1} \mid S P C\left(n-1, p_{1}, p_{2}\right)\right)+\frac{20}{3}(n-1)+\frac{35}{6}\right] \\
& +p_{2}\left[r\left(u_{n-1} \mid S P C\left(n-1, p_{1}, p_{2}\right)\right)+\frac{25}{6}(n-1)+\frac{35}{6}\right] \\
& +\left(1-p_{1}-p_{2}\right)\left[r\left(u_{n-1} \mid S P C\left(n-1, p_{1}, p_{2}\right)\right)+\frac{15}{2}(n-1)+\frac{35}{6}\right]
\end{aligned}
$$

By applying the expectation operator to the above equation, we obtain

$$
U_{n}=U_{n-1}+\left(\frac{15}{2}-\frac{5}{6} p_{1}-\frac{10}{3} p_{2}\right) n+\frac{5}{6} p_{1}+\frac{10}{3} p_{2}-\frac{5}{3}
$$

And $U_{1}=E\left(r\left(u_{1} \mid S P C\left(1, p_{1}, p_{2}\right)\right)\right)=\frac{35}{6}$, using the above recurrence relation, we have

$$
U_{n}=\left(\frac{15}{4}-\frac{5}{12} p_{1}-\frac{5}{3} p_{2}\right) n^{2}+\left(\frac{25}{12}+\frac{5}{12} p_{1}+\frac{5}{3} p_{2}\right) n
$$

From (4),

$$
\begin{aligned}
& E\left(K f\left(S P C\left(n+1, p_{1}, p_{2}\right)\right)=\right. \\
& =E\left(K f\left(S P C\left(n, p_{1}, p_{2}\right)\right)\right)+5\left[\left(\frac{15}{4}-\frac{5}{12} p_{1}-\frac{5}{3} p_{2}\right) n^{2}+\right. \\
& \left.\quad\left(\frac{25}{12}+\frac{5}{12} p_{1}+\frac{5}{3} p_{2}\right) n\right]+\frac{175}{6} n+\frac{35}{2}
\end{aligned}
$$

and $E\left(K f\left(S P C\left(1, p_{1}, p_{2}\right)\right)\right)=\frac{35}{2}$.

Using the above recurrence relation, we have

$$
\begin{array}{r}
E\left(K f\left(S P C\left(n, p_{1}, p_{2}\right)\right)\right)=\left(\frac{25}{4}-\frac{25}{36} p_{1}-\frac{25}{9} p_{2}\right) n^{3}+\left(\frac{25}{12} p_{1}+\frac{25}{3} p_{2}+\frac{125}{12}\right) n^{2} \\
-\left(\frac{25}{18} p_{1}+\frac{50}{9} p_{2}-\frac{5}{6}\right) n .
\end{array}
$$

Specially, by taking $\left(p_{1}, p_{2}\right)=(1,0),(0,1)$ or $(0,0)$, respectively, and Theorem 2.3, we have

Corollary 2.4. ([8]) The Kirchhoff indices of the spiro meta-chain $M_{n}$, the spiro orthochain $O_{n}$ and the spiro para-chain $P_{n}$ are

$$
\begin{aligned}
& K f\left(M_{n}\right)=\frac{50}{9} n^{3}+\frac{25}{2} n^{2}-\frac{5}{9} n \\
& K f\left(O_{n}\right)=\frac{125}{36} n^{3}+\frac{75}{4} n^{2}-\frac{85}{18} n \\
& K f\left(P_{n}\right)=\frac{25}{4} n^{3}+\frac{125}{12} n^{2}+\frac{5}{6} n .
\end{aligned}
$$




\subsection{A relation between $E(K f(P P C))$ and $E(K f(S P C))$}

Since a spiro chain can be obtained from a polyphenyl chain by squeezing off its cut edges, it is straightforward by Rayleigh short-cut principle in the classical theory of electricity that the Kirchhoff index of the spiro chain is less than the polyphenyl chain. In fact, a relation between the Kirchhoff indices of a polyphenyl chain and its corresponding spiro chain obtained by squeezing off its cut edges was given in [8]. Here, we can also obtain a relation between the expected values of their Kirchhoff indices of the random polyphenyl chain $P P C\left(n, p_{1}, p_{2}\right)$ and the random spiro chain $S P C\left(n, p_{1}, p_{2}\right)$ with the same probabilities $p_{1}$ and $p_{2}$ from Theorems 2.1 and 2.3.

Theorem 2.5. For a random polyphenyl chain $P P C\left(n, p_{1}, p_{2}\right)$ and a random spiro chain $S P C\left(n, p_{1}, p_{2}\right)$ with $n$ hexagons, the expected values of their Kirchhoff indices are related as

$$
50 E\left(K f\left(P P C\left(n, p_{1}, p_{2}\right)\right)\right)=72 E\left(K f\left(S P C\left(n, p_{1}, p_{2}\right)\right)\right)+300 n^{3}-350 n^{2}-335 n .
$$

Theorem 2.5 also shows that the expected value of Kirchhoff index of the random spiro chain is less than the random polyphenyl chain. In fact, for $n \geq 2, E(K f(S P C(n$, $\left.\left.\left.p_{1}, p_{2}\right)\right)\right)<\frac{25}{36} E\left(K f\left(P P C\left(n, p_{1}, p_{2}\right)\right)\right)$. The reason is quite obvious. Dividing both sides of the equation in Theorem 2.5 yields

$$
E\left(K f\left(P P C\left(n, p_{1}, p_{2}\right)\right)\right)=\frac{36}{25} E\left(K f\left(S P C\left(n, p_{1}, p_{2}\right)\right)\right)+6 n^{3}-7 n^{2}-\frac{67}{10} n
$$

and it is easily seen that for $n \geq 2,6 n^{3}-7 n^{2}-\frac{67}{10} n>0$.

\subsection{The average value of the Kirchhoff index}

Let $\overline{\mathcal{G}}_{n}$ is the set of all polyphenyl chains with $n$ hexagons. The average value of the Kirchhoff indices with respect to $\overline{\mathcal{G}}_{n}$ is

$$
K f_{a v r}\left(\overline{\mathcal{G}}_{n}\right)=\frac{1}{\left|\overline{\mathcal{G}}_{n}\right|} \sum_{G \in \overline{\mathcal{G}}_{n}} K f(G) .
$$

In order to obtain the average value of the Kirchhoff indices with respect to $\overline{\mathcal{G}}_{n}$, we only need to take $p_{1}=p_{2}=\frac{1}{3}$ in the random polyphenyl chain $P P C\left(n, p_{1}, p_{2}\right)$, i.e., the average value of the Kirchhoff indices with respect to $\overline{\mathcal{G}}_{n}$ is just the expected value of the Kirchhoff index of the random polyphenyl chain $\operatorname{PPC}\left(n, p_{1}, p_{2}\right)$ for $p_{1}=p_{2}=\frac{1}{3}$. From Theorem 2.1, we have

Theorem 2.6. The average value of the Kirchhoff indices with respect to $\overline{\mathcal{G}}_{n}$ is

$$
K f_{\text {avr }}\left(\overline{\mathcal{G}}_{n}\right)=\frac{40}{3} n^{3}+13 n^{2}-\frac{53}{6} n .
$$

Similarly, let $\mathcal{G}_{n}$ is the set of all spiro chains with $n$ hexagons. The average value of the Kirchhoff indices with respect to $\mathcal{G}_{n}$ is

$$
K f_{\text {avr }}\left(\mathcal{G}_{n}\right)=\frac{1}{\left|\mathcal{G}_{n}\right|} \sum_{G \in \mathcal{G}_{n}} K f(G) .
$$


And the average value of the Kirchhoff indices with respect to $\mathcal{G}_{n}$ is just the the expected value of the Kirchhoff index of the random spiro chain $S P C\left(n, p_{1}, p_{2}\right)$ for $p_{1}=p_{2}=\frac{1}{3}$. From Theorem 2.3, we have

Theorem 2.7. The average value of the Kirchhoff indices with respect to $\mathcal{G}_{n}$ is

$$
K f_{\text {avr }}\left(\mathcal{G}_{n}\right)=\frac{275}{54} n^{3}+\frac{125}{9} n^{2}-\frac{40}{27} n .
$$

Acknowledgments. The authors would like to thank the anonymous referees for their valuable comments and suggestions on the original manuscript.

\section{References}

[1] D. Babić, D. J. Klein, I. Lukovits, S. Nikolić, N. Trinajstić, Resistance- distance matrix: A computational algorithm and its application, Int. J. Quantum Chem. 90 (2002), 166-176.

[2] Y. Bai, B. Zhao, P. Zhao, Extremal Merrifield-Simmons index and Hosoya index of polyphenyl chains, MATCH Commun. Math. Comput. Chem. 62 (2009), 649-656.

[3] R. B. Bapat, I. Gutman, W. J. Xiao, A simple method for computing resistance distance, Z. Naturforsch 58a (2003), 494-498.

[4] D. Bonchev, E. J. Markel, A. H. Dekmezian, Long chain branch polymer chain dimensions: application of topology to the Zimm-Stockmayer model, Polymer 43 (2002), 203-222.

[5] X. Chen, B. Zhao, P. Zhao, Six-membered ring spiro chains with extremal Merrifild-Simmons index and Hosoya index, MATCH Commun. Math. Comput. Chem. 62 (2009), 657-665.

[6] H. Deng, On the minimal Kirchhoff indices of graphs with a given number of cut edges, MATCH Commun. Math. Comput. Chem. 63 (2010), 171-180.

[7] H. Deng, Wiener indices of spiro and polyphenyl hexagonal chains, Mathematical and Computer Modelling 55 (2012), 634-644.

[8] H. Deng, Z. Tang, Kirchhoff indices of spiro and polyphenyl hexagonal chains, accepted by Util. Math.

[9] T. Došlić, F. Måløy, Chain hexagonal cacti: Matchings and independent sets, Discrete Math. 310 (2010), 1676-1690.

[10] T. Došlić, M. S. Litz, Matchings and independent sets in polyphenylene chains, MATCH Commun. Math. Comput. Chem. 67 (2012), 313-330.

[11] D. R. Flower, On the properties of bit string-based measures of chemical similarity, J. Chem. Inf. Comput. Sci. 38 (1998), 379-386.

[12] Q. Guo, H. Deng, The extremal Kirchhoff index of a class of unicyclic graphs, MATCH Commun. Math. Comput. Chem. 61 (2009), 713-722.

[13] D. J. Klein, M. Randić, Resistance distance, J. Math. Chem. 12 (1993), 81-95.

[14] D. J. Klein, Graph geometry, graph metrics and Wiener, MATCH Commun. Math. Comput. Chem. 35 (1997), 7-27.

[15] D. J. Klein, H. Y. Zhu, Distances and volumina for graphs, J. Math. Chem. 23 (1998), 179-195.

[16] D. J. Klein, Resistance-distance sum rules, Croat. Chem. Acta 75 (2002), 633-649.

[17] Q. R. Li, Q. Yang, H. Yin, S. Yang, Analysis of by-products from improved Ullmann reaction using TOFMS and GCTOFMS, J. Univ. Sci. Technol. China 34 (2004), 335-341.

[18] J. L. Palacios, Closed-form formulas for Kirchhoff index, Int. J. Quantum Chem. 81 (2001), $135-140$. 
[19] J. L. Palacios, Resistance distance in graphs and random walks, Int. J. Quantum Chem. 81 (2001), 29-33.

[20] S. Tepavčević, A. T. Wroble, M. Bissen, D. J. Wallace, Y. Choi, L. Hanley, Photoemission studies of polythiophene and polyphenyl films produced via surface polymerization by ionassisted deposition, J. Phys. Chem. B 109 (2005), 7134-7140.

[21] Y. J. Yang, X. Y. Jiang, Unicyclic graphs with extremal Kirchhoff index, MATCH Commun. Math Comput. Chem. 60 (2008), 107-120.

[22] Y. Yang, H. Zhang, Kirchhoff index of linear hexagonal chains, Int. J. Quantum Chem. 108 (2008), 503-512.

[23] W. Yang, F. Zhang, Wiener index in random polyphenyl chains, MATCH Commun. Math. Comput. Chem. 68 (2012), 371-376.

[24] H. Zhang, Y. Yang, Resistance distance and Kirchhoff index in circulant graphs, Int. J. Quantum Chem. 107 (2007), 330-339.

[25] W. Zhang, H. Deng, The second maximal and minimal Kirchhoff indices of unicyclic graphs, MATCH Commun. Math. Comput. Chem. 61 (2009), 683-695.

[26] H. Zhang, X. Jiang, Y. Yang, Bicyclic graphs with extremal Kirchhoff index, MATCH Commun. Math. Comput. Chem. 61 (2009), 697-712.

[27] P. Zhao, B. Zhao, X. Chen, Y. Bai, Two classes of chains with maximal and minimal total $\pi$-electron energy, MATCH Commun. Math. Comput. Chem. 62 (2009), 525-536. 